\title{
Mass hierarchy and CP violation at existing facilities
}

\section{Jennifer Thomasj ${ }^{* i}$}

Author University College London

E-mail: jennifer.thomas@ucl.ac.uk

The NuMI neutrino beam at FNAL offers a substantial reach in neutrino mass hierarchy and CP violation. It will come online after an upgrade in Fall of 2013 and will provide the world's most intense neutrino beam. The baseline offered by its location, provides the neutrino oscillations with matter effects, and an intensity sufficient to study $\mathrm{CP}$ violation with better precision than anywhere else in the coming decade. New ideas are coming forward about how best to exploit this opportunity, using both Liquid Argon and water Cherenkov technology

XV Workshop on Neutrino Telescopes,

11-15 March 2013

Venice, Italy

* Speaker.

${ }^{\dagger}$ A footnote may follow. 


\section{Introduction}

Collecting the neutrinos presently pouring out of the NuMI beam line at Fermi National Accelerator Laboratory (FNAL) can provide a very high pedestal on which to base the next generation of long baseline neutrino events presently being planned in the US (LBNE) and in Europe (LBNO). Given the paucity of neutrino interactions at long baselines presently existing, and the length of time expected to mount any one of the next generation LB experiments (at least 10-12 years), the NuMI beam line can provide a unique opportunity to get started on the measurements of the Mass Hierarchy $(\mathrm{MH})$, indeed already happening at the NOvA experiment, and the CP violating angle $\delta_{C P}$. While measurement of the $\mathrm{MH}$ relies on a rather long baseline, the measurement of $\delta_{C P}$ benefits from the increased flux at shorter distances than those being designed for the future. Furthermore, the beam power expected from the next generation of experiments is only moderately increased compared to the NuMI's 700kW. This limitation on beam power points to the need for much larger detectors in the future than have presently been envisaged (on the order of a Mega-ton) in order to make precision studies of the oscillation parameters. Precision detectors, such as Liquid Argon TPCs may not be enough: a combination of LAr for exquisite reconstruction and water Cherenkov for huge statistics will likely be needed in the longer term. Focussing resources now on existing neutrino beams, and emphasizing the reusability of the detector components, can vastly reduce the time needed to get to the answers.

\section{E-NuMI and the specific options}

A group was formed in January to look at the potential options for further exploitation of the NuMI beam. There were about eighty people signed up to this group at its peak. There were three real options put forward

1. More NOvA Detector. The NOvA hall has room for a further $4 \mathrm{kt}$ of detector, while there were only funds to build $14 \mathrm{kt}$. The factories could remain in operation and the further detector mass could be produced.

2. GLADE (now RADAR) at Ash River. This would be a 5-6kt Liquid Argon detector located in the extra space in the Ash River NOvA site.

3. CHIPS in the Wentworth $2 \mathrm{~W}$ pit. This would be eventually a 100kt water Cherenkov detector submerged from the surface of a mine pit which is intersected by the NuMI beam. It would start small and grow as funds were available.

Since the conference, two distinct new collaborations have formed : CHIPS and RADAR.

\section{The NuMI Beam}

The MINOS, NOvA and MINERvA experiments each have extensive simulations of the NuMI beam. By taking advantage of the fact that neutrino production from decaying hadrons is isotropic in the center of mass frame, and that the existing simulations store neutrino parent information, we can reweight the existing MC to give a neutrino flux at any location in the beam [1]. Furthermore we 


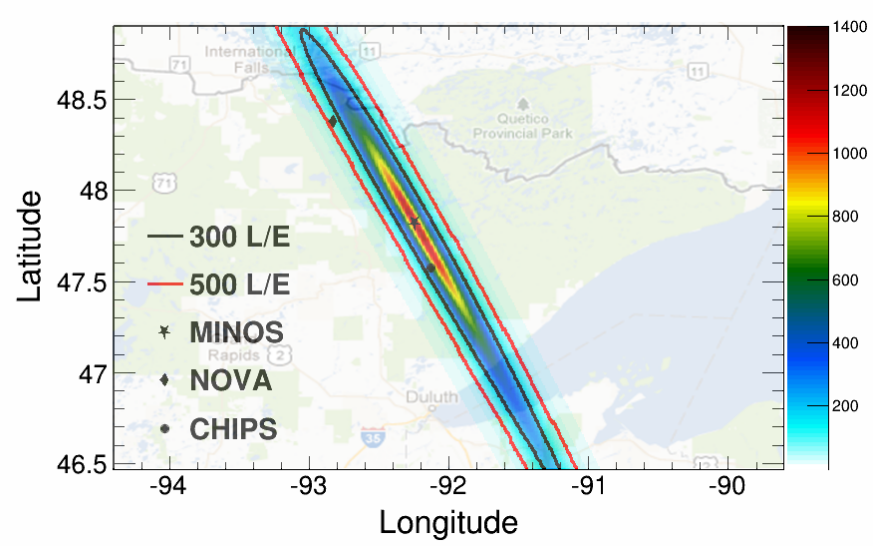

Figure 1: A map of potential neutrino event rates, assuming no oscillations, between $0-30 \mathrm{GeV}$ for an exposure of $1 \mathrm{kton}$-year. Contours show lines of constant $\mathrm{L} / \mathrm{E}$ where $\mathrm{L}$ is the distance from the hypothetical detector to the NuMI target and $\mathrm{E}$ is the peak energy of the reweighted neutrino spectrum

scan over a region of interest to construct a map of flux characteristics such as peak energy. Cross section and oscillation information can also be included to give a clear, intuitive impression of the oscillation sensitivity at a given location. Figure 1 shows the computed $v_{\mu}$-CC event rate integrated over all energies for various locations in northern Minnesota. Figure 2 shows the predicted $v_{\mu}$-CC event energy spectra at different detector locations in northern Minnesota.
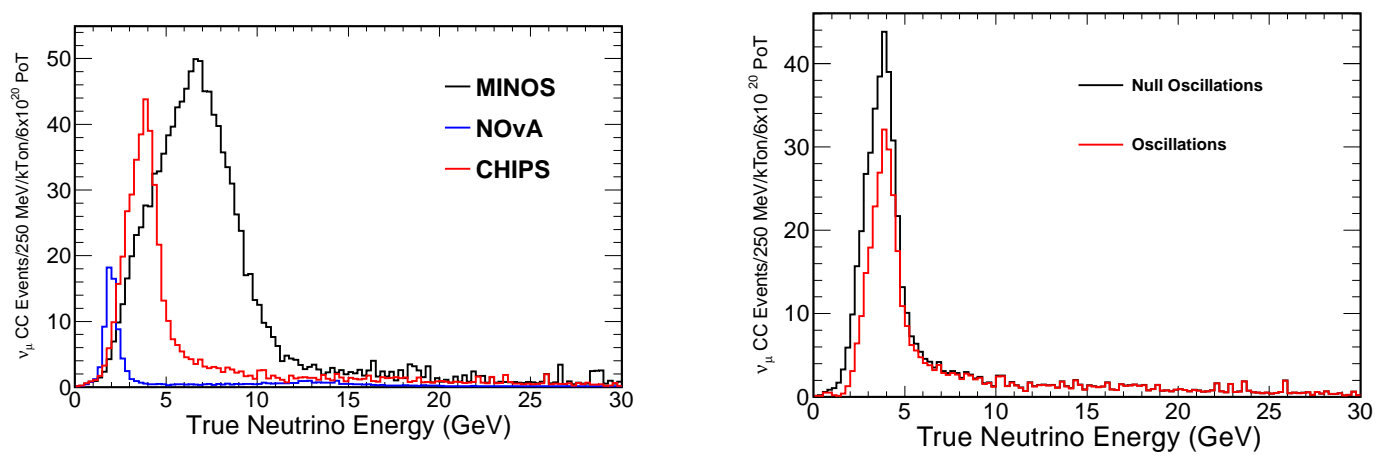

Figure 2: (Left) True energy distribution of $v_{\mu}$-CC events at the MINOS, NOvA, and CHIPS far detector locations, assuming no oscillations and 1 kton-year of exposure. (Right) The true energy distribution of $v_{\mu}$-CC events that would be seen at CHIPS in one kiloton year with (red) and without (black) neutrino oscillations.

\section{More NOvA}

The baseline exposure for the NOvA experiment assumes a $14 \mathrm{kt}$ detector, $700 \mathrm{~kW}$ NuMI beam power, and 6 years of running. Doubling the NOvA exposure would require a relatively modest investment, have low risks, and would leverage the substantial investments made in the NuMI beam, the Ash River laboratory site, and the setup of the NOvA production factories. Construction of the NOvA detector is underway; $1 / 4$ of the detector is in place and is being filled with scintillator. An 

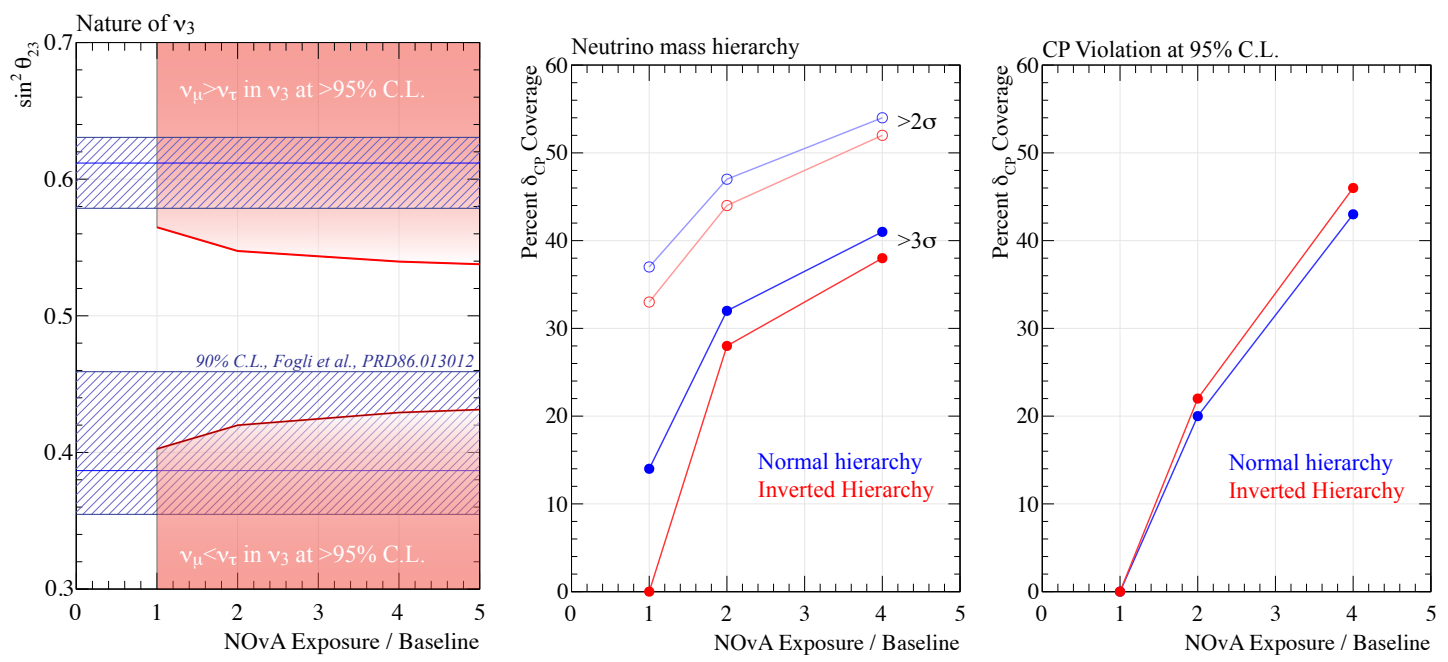

Figure 3: Left: extended reach for resolving the nature of $v_{3}$ relative to the current knowledge of $\sin ^{2} \theta_{23}$ following Neutrino 2012. Middle: the percent of $\delta_{\mathrm{CP}}$ values for which NOvA can resolve the neutrino mass hierarchy at 2 and $3 \sigma$ C.L.(left). Right: The percent of $\delta_{\mathrm{CP}}$ values for which NOvA can establish CP violation at $95 \%$ C.L. or better.

increase in exposure of $2.1 \times$ can be realized by increasing the detector mass to $18 \mathrm{kt}$ and extending the run to 10 years. This run plan uses all of the available space in the laboratory and recognizes the schedule realities of next-generation projects. If construction of the NOvA detector is continued without interruption, the cost of the additional mass would be $\$ 6 \mathrm{M} / \mathrm{kt}$. A conservative upper limit which assumes that current construction ends and that all the start-up costs must be paid again on future construction raises this figure to $\$ 9 \mathrm{M} / \mathrm{kt}$.

Additional exposure could follow in a second phase and is motivated by the LBNE reconfiguration report [2] which found Ash River to be the site with maximum $\mathrm{CP}$ reach assuming that the mass hierarchy is resolved by the experiments planned for this decade (eg. NOvA, Pingu, Daya Bay II). A $5 \mathrm{kt}$ liquid argon TPC at the Ash River site, either in the NOvA laboratory or in a new facility which reuses the infrastructure supporting the NOvA laboratory, effectively increases the NOvA exposure by a factor of 4 given the improved performance of liquid argon detectors.

Figures 3 outline what is possible with additional exposure. The left figure shows the extended reach for resolving the nature of $v_{3}$ relative to the current knowledge of $\sin ^{2} \theta_{23}$ following Neutrino 2012. NOvA's baseline measurement covers $64 \%$ of the currently allowed $90 \%$ C.L. region at $95 \%$ C.L. or better. With $2 \times$ the exposure this increases to $75 \%$ and $80 \%$ for $4 \times$. The middle figure shows the improvement in mass hierarchy resolution. With additional exposure, a significant amount of coverage is obtained at $>3 \sigma$ over the baseline experiment. Finally, NOvA's reach for $\mathrm{CP}$ violation increases rapidly with exposure in the right figure. NOvA's baseline exposure enables a first measurement of $\delta_{\mathrm{CP}}$ but the precision will not be enough to establish CP violation. CP violation can be established with $95 \%$ C.L. for $20 \%$ of the $\delta_{\mathrm{CP}}$ space for $2 \times$ the exposure, increasing to $45 \%$ for $4 \times$ the exposure. 


\section{GLADE/RADAR: R\&D Argon Detector at Ash River}

The R\&D Argon Detector at Ash River (RADAR) project is aimed at enhancing the full Homestake LBNE project in four critical sectors: physics reach; timeline; cost; and the development of international partnerships. LBNE is a planned high-statistics neutrino oscillation experiment that will use a new neutrino beam line at Fermilab. In its full configuration, LBNE will operate a 34 kton liquid argon time-projection chamber (LAr TPC) at the 4850' level of the Homestake Mine in Lead, South Dakota [3].

The RADAR project consists of a $6 \mathrm{kton}$ (4.6 kton fiducial) LAr TPC sited in the existing NOvA Far Detector building at Ash River, Minnesota. The detector would record neutrino interactions from the $2 \mathrm{GeV}$ narrow band neutrino beam from the recently upgraded $700 \mathrm{~kW}$ NuMI source at Fermilab. With this arrangement, results from RADAR would allow for further optimization of LBNE to maximize its physics program reach, while significantly extending the physics capabilities of the NuMI beam-based NOvA program operating at Fermilab and Ash River.

The RADAR sensitivity studies were carried out within the NOvAcode framework where we assumed: NuMI operates at $700 \mathrm{~kW}$ in the medium energy beam configuration; the RADAR liquid argon detector has a fiducial mass of 5 kton and begins operation in 2019, running for 5 years; NOvA has a fiducial mass of 14 kton and operates for 10 years, including 6 years from 2014 to 2019 for its nominal run, and 4 years from 2020 to 2023, concurrently with RADAR data taking; The T2K long-baseline experiment completes its projected 6-year run during this decade. We further assume equal running time in neutrino and antineutrino modes for NOvA and RADAR, but exclusive neutrino-mode running for $\mathrm{T} 2 \mathrm{~K}$.

The NOvA contribution is calculated using the information in Ref. [4]. The T2K contribution is calculated using the information in Ref. [5]. The sensitivities from a LAr detector at Ash River are taken as equivalent to using NOvA detector technology with three times the mass of the LAr detector [6].

Figures 4 and 5(left) demonstrate the significant enhancement of the mass hierarchy and CP violation reach of the Ash River program provided by RADAR. The hierarchy significance surpasses $4.5 \sigma$ at the most favorable $\delta$ values and is greater than $95 \%$ C.L. for over half the $\delta$ range. In the other half of the $\delta$ range, the hierarchy information gained from RADAR is correlated with the $\delta$ information. The reach for CP violation is also improved, with RADAR offering the first ever $95 \%$ C.L. sensitivity to $\mathrm{CP}$ violation. The near-maximal mixing implied by the current measurements of $\theta_{23}$ can also be tested at a level well beyond current limits. RADAR will reduce the uncertainty on $\sin ^{2} 2 \theta_{23}$ by $40 \%$ relative to NOvA and can see deviations from maximal mixing at the $95 \%$ C.L. all the way up to $\sin ^{2} 2 \theta_{23}=0.994$. Furthermore, the flavor structure of the $v_{3}$ state, characterized by the octant of $\theta_{23}$, can be probed in RADAR beyond $\sin ^{2} 2 \theta_{23}=0.99$ at $95 \%$ C.L., as shown in Fig. 5(right).

\section{CHIPS : CHerenkov detectors In mine PitS}

It is likely that water is the only way we will get to a mega-ton detector scale. Not only is the medium very cheap, but also the instrumentation is more cost-effective, needing only of order $10 \%$ of the surface area of the detector to be instrumented. The idea of CHIPS is to use the surrounding 

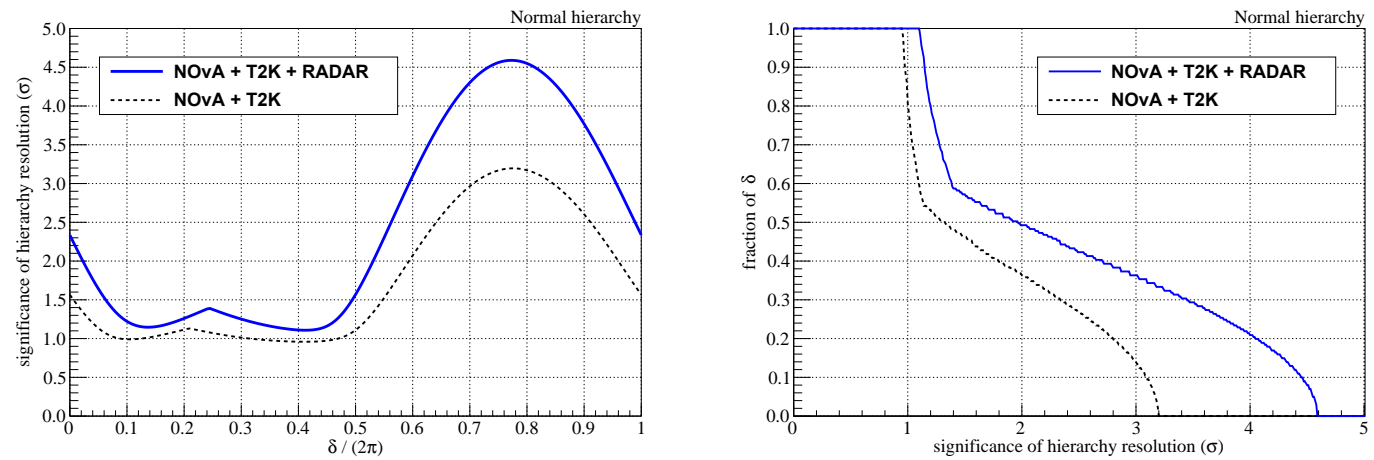

Figure 4: The significance of hierarchy resolution for NOvA+T2K alone (black dashed) and with RADAR added (blue solid), shown both as a function $\delta$ (left) and in terms of the fraction of $\delta$ values covered at a given confidence level. Normal hierarchy and maximal mixing are used.
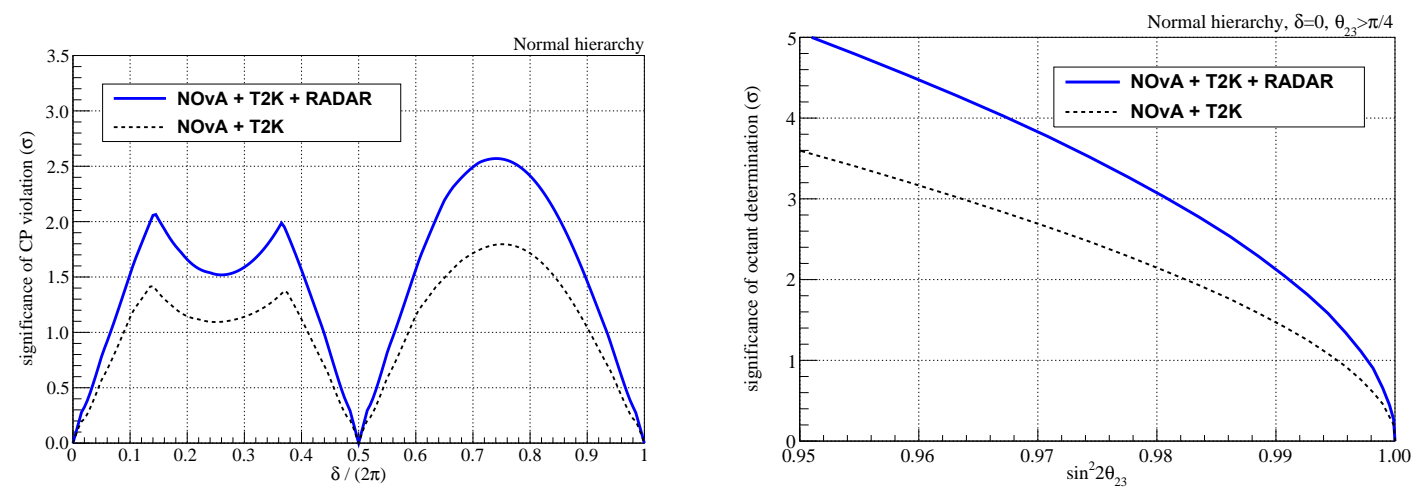

Figure 5: Left: the significance of $\mathrm{CP}$ violation for $\mathrm{NO} v \mathrm{~A}+\mathrm{T} 2 \mathrm{~K}$ alone (black dashed) and with RADAR added (blue solid), shown as a function $\delta$ (left). Normal hierarchy and maximal mixing are used. Right: the significance with which the $\theta_{23}$ octant can be determined as a function of the true value of $\sin ^{2} 2 \theta_{23}$, shown for NOvA + T2K alone (black dashed) and with RADAR added (blue solid). $\theta_{13}$ is assumed known to $5 \%$ and the uncertainty on $\sin ^{2} 2 \theta_{23}$ is taken from NOvA [4] and T2K projections [5]. Normal hierarchy, $\delta=0$, and true $\theta_{23}>0$ are assumed. RADAR reduces the inaccessible range of $\sin ^{2} 2 \theta_{23}$ for a given desired confidence level by approximately a factor of two.

water as the mechanical support for a light-tight bag which will be submerged and filled with purified water. The choice of location requires neither expensive mechanical structures nor an underground cavern, two of the dominant expenses associated with traditional neutrino detector designs. CHIPS will comprise a mechanical truss frame surrounded by a light and water tight liner. The liner will be an opaque polymer membrane, like ones used in the geomembrane and roofing industry, and filled with purified water. The space frame will be made of hollow pipes, similar to those used in the roof structure in Figure 6(right), which can be filled with water or air to enable submersion or flotation.

The PMTS would likely be contained inside watertight plastic housings with HV sent down to and signals sent up from the detector. The PMTs for LBNE have been designed to withstand $60 \mathrm{~m}$ hydrostatic pressure, and so while the watertight housings are not completely necessary, for safety 
we think designing a generic watertight housing is important for the robustness of the detector, especially given that these PMTs will have to be raised and lowered several times while the detector is being instrumented. Common marina technology would be used to keep floating structures free of winter ice. The purification system for such a set up can be tailored for the level of water clarity needed. On the order of 6000 PMTs will be needed for the 10kt prototype. The baseline photodetectors could be similar to currently available 10"-12" PMTs, which have been designed to withstand more than $60 \mathrm{~m}$ hydrostatic pressure[3] although it is hoped other possibilities become available during the course of the incremental construction.

Individual photodetector panels on the surface of the cylinder will be mounted on the space frame extending $1-2 \mathrm{~m}$ perpendicular to the instrumented plane as shown in Figure 6. Moulded acrylic housings encompass the PMTSs while providing a secure mounting system.

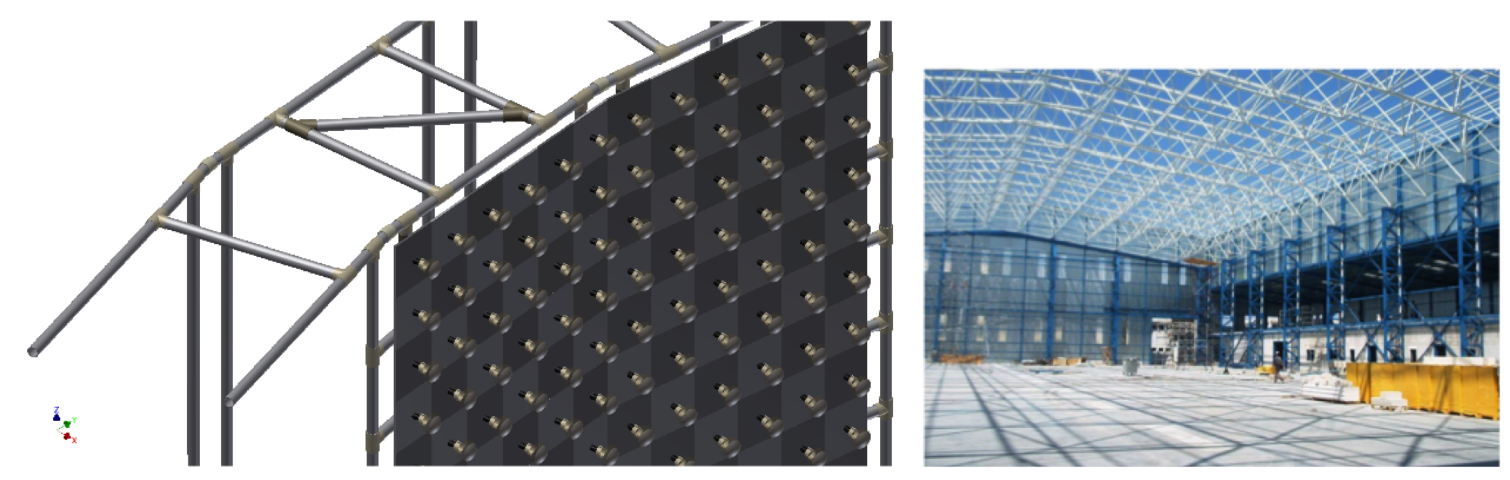

Figure 6: Panels supporting PMTs (left) and example of structure (right).

The minimum operation depth of $40 \mathrm{~m}$ (and therefore the detector height of $20 \mathrm{~m}$ ) is set by the rate of cosmic ray muons, which rises to impractical levels at shallower depths. Even at 40m, the rate will be substantial and beam spills containing such background muons must be tagged for removal. Therefore a fraction of the photodetectors will be arranged to point outwards into a $2 \mathrm{~m}$-thick veto volume along the top and side of the cylinder, where they will reliably detect Cherenkov light from background muons. This veto volume also provides room for the support framework, and is separated from the active volume by the non-watertight light barrier made up of the individual PMT panels.

Outside the entire detector is the reinforced polymer membrane (liner) that blocks outside light and isolates the pure water inside from the pit water. Such a membrane was selected for the proposed GRANDE experiment[7] and is one of many options used in the geomembrane and roofing material industries for blocking water over large areas. For CHIPS, the liner will be maintained in a cylindrical shape between several large stiff rings, connected to each other and moored in the lake using strong support cables. Such rings and mooring systems are routinely used for construction of net cages in the aquaculture industry, including deployments up to $64 \mathrm{~m}$ diameter in open sea conditions[?]. See the schematic in Figure 7.

The perfect candidate pit, $60 \mathrm{~m}$ deep, $707 \mathrm{~km}$ from FNAL, called Wentworth $2 \mathrm{~W}$ in Northern Minnesota, is found at a very optimal off-axis angle of $7 \mathrm{mrad}$, delivering the optimal trade off between event rate and background rejection, as shown in Figure 8. 

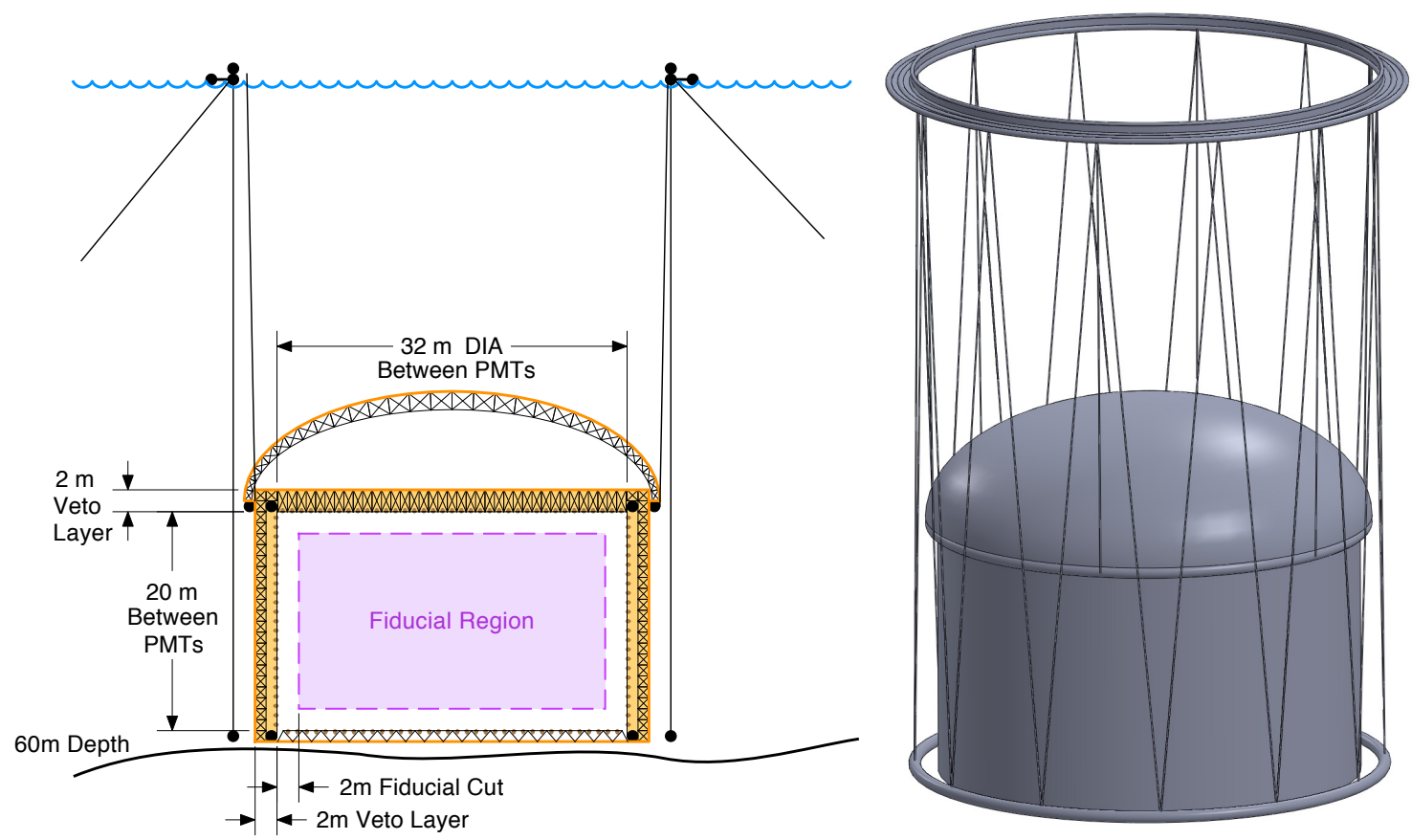

Figure 7: Full schematic showing CHIPS-10 prototype.
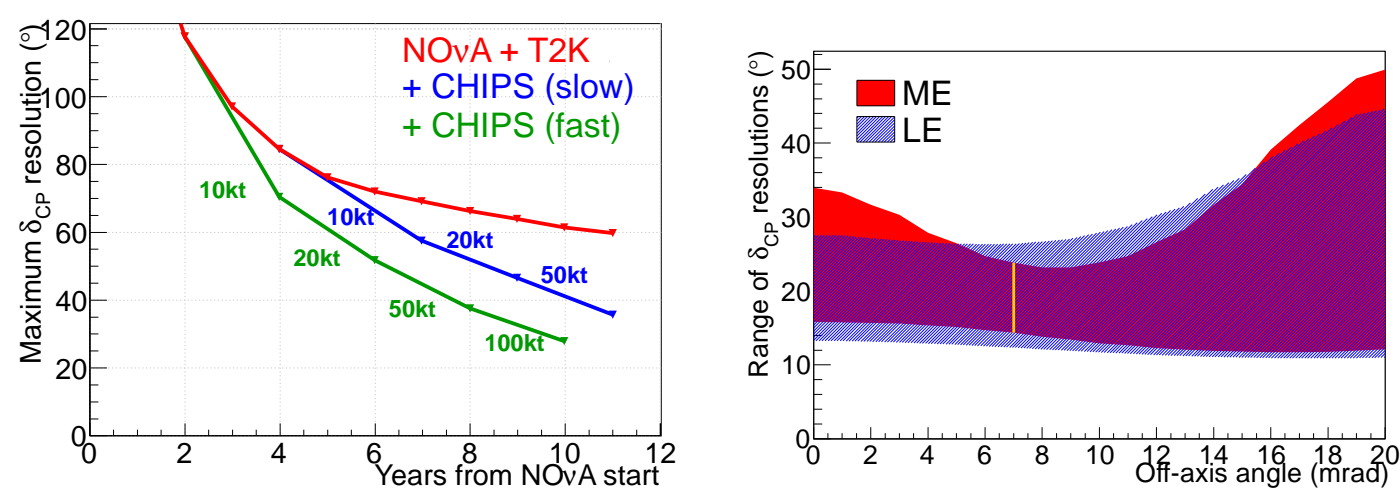

Figure 8: (Left) Impact of a phased CHIPS program on $\delta_{C P}$ resolution. (Right) $\delta_{C P}$ resolution band for offaxis angles from 0 to $20 \mathrm{mrad}$, for the ME and LE beams. The orange line at an angle of $7 \mathrm{mrad}$ corresponds to the position of the Wentworth pit.

CHIPS would provide complementary information on $\delta_{C P}$ to NOvA owing to the broader beam spectrum at $7 \mathrm{mrad}$. By the end of the decade, it could be possible to push the error on $\delta_{C P}$ down to between $15-28^{\circ}$, and this will not be systematics limited. Figure 8(left) shows how adding a phased-CHIPS detector in the NuMI beamline improves the $\delta_{C P}$ resolution over the default configuration of NOvA and T2K only. Two approaches are shown; a fast track approach of building a 10 kton detector two years after NOvA starts data taking and increasing this to 20,50 and $100 \mathrm{kton}$ every subsequent two years. The other is a slower-track approach. 


\section{Summary}

In summary, a modest investment to extend the NOvA exposure to $2 \times$ its baseline through a combination of detector mass and running time would yield qualitative improvements in the experiment's hierarchy and CP violation reach. A 5 kt liquid argon TPC at the Ash River site could extend the physics reach further in a second phase. These extensions would leverage the investments made in the NOvA factories, the Ash River laboratory, and the NuMI beam. Valuable knowledge gained from LAr TPC construction and operations with neutrino interactions, along with the reusability of components in the LBNE full-scale Far Detector, can significantly offset the projected costs of the RADAR proposal. RADAR provides continuity of a vibrant US longbaseline neutrino physics program, potentially improving the sensitivity to the mass hierarchy to more than $4.5 \sigma$ at the most favorable $\delta$ and the reach for CP violation to $95 \%$ C.L. across a quarter of the $\delta$ range. The complementary CHIPS detector built incrementally over the next decade can provide important new results. The CHIPS detector could then be redeployed off-axis in the LBNE beam, to improve the overall reach of the LBNE project. Even with a 10kt LAr LBNE detector, $\delta_{C P}$ angles as small as $10-15^{\circ}$ would be within reach after 10 years of running on top of the baseline of 6 years of running in NuMI. This is to be compared with a neutrino factory, whose best $\delta_{C P}$ reach is estimated to be about $5^{0}$. This situation is summarized in Figure 9. The message from this study is that no neutrino should be wasted: each one can add to the overall world knowledge.

\section{References}

[1] Neutrino Beam Simulation Using PAW with Weighted Monte Carlo. Milburn, R., Unpublished, MINOS-doc-109 (1995).

[2] J. Appel et al, "Physics Working Group Report to the LBNE Reconfiguration Steering Committee", http://www.fnal.gov/directorate/lbne_reconfiguration/files/LBNE-Reconfiguration-PhysicsWGReport-August2012.pdf

[3] LBNE Conceptual Design Report (LBNE Collaboration), http://lbne.fnal.gov/reviews/CD1-CDR.shtml (2012).

G. L. Fogli, E. Lisi, A. Marrone, D. Montanino, A. Palazzo and A. M. Rotunno, Phys. Rev. D 86, 013012 (2012), arXiv:1205.5254 [hep-ph].

[4] R. Patterson, "The NOvA Experiment: Status and Outlook", Nucl. Phys. B (Proc. Suppl.) 235-236, 151-157 (2013); arXiv:1209.0716 (2012).

[5] T. Nakaya, "New Results from T2K”, Nucl. Phys. B (Proc. Suppl.) 235-236, 97-104 (2013); presentation at http://kds.kek.jp/getFile.py/access?contribId=14\&sessionId=7\&resId=0\&materialId=slides\&confId=9151 (2012).

[6] M. Messier for NOvA, "Extending the NOvA Physics Program," Snowmass whitepaper, http://if-neutrino.fnal.gov/whitepapers/messier-nova.pdf (2013).

[7] The GRANDE Detector, Sobel, H. et al.,Nucl. Phys. Proc. Suppl, 14A, 125-142, (1990)

[8] Aqualine, Norway, url = http://www.aqualine.no/en/news-and-press/news 

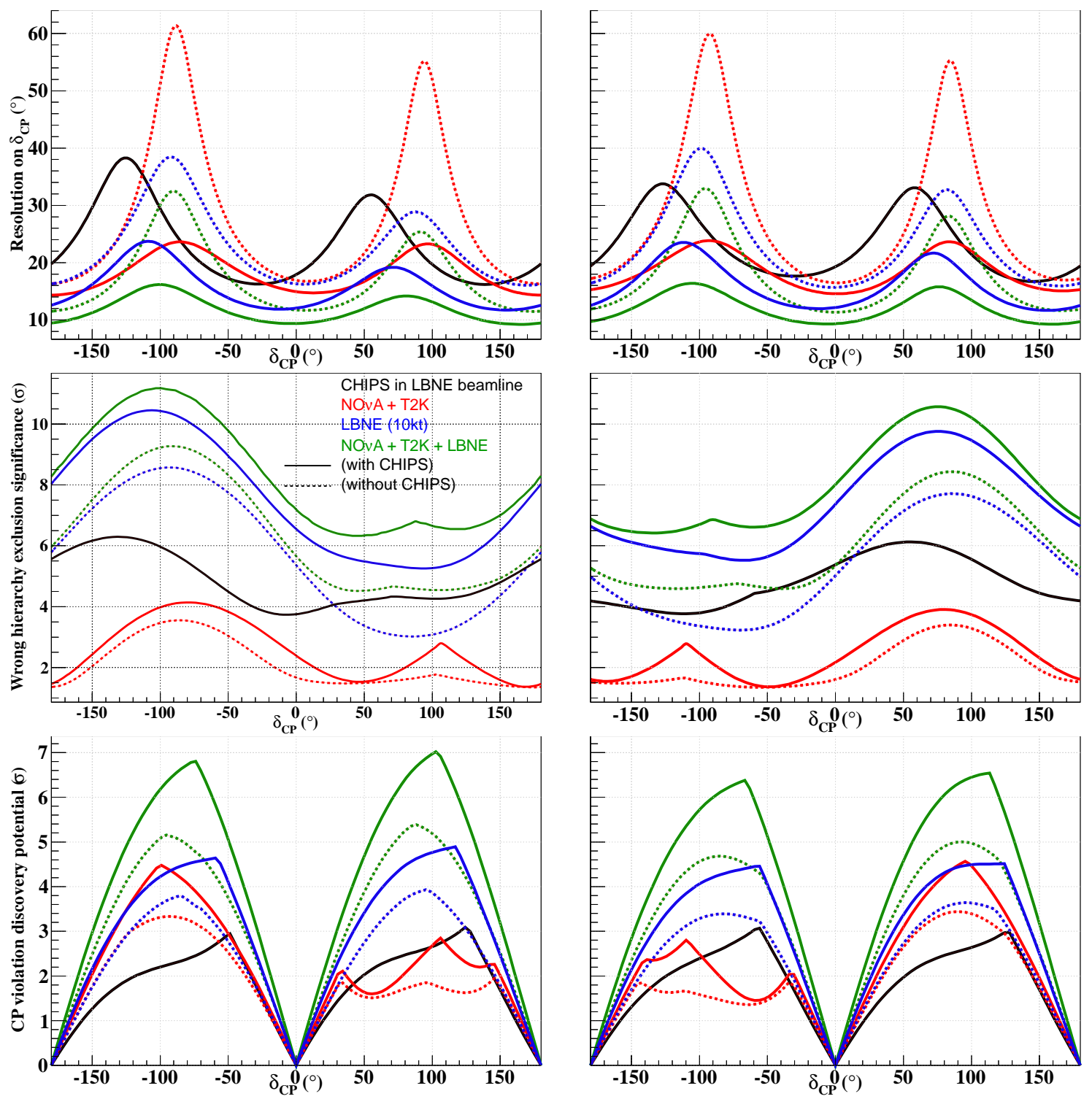

Figure 9: Physics reach in the Normal Hierarchy (left) and Inverted Hierarchy (right), for NOvA+T2K, $10 \mathrm{kton}$ LAr LBNE, and CHIPS in the LBNE beam at $20 \mathrm{mrad}$. (Top) $\delta_{C P}$ resolutions. (Middle) The significance of excluding the wrong hierarchy. (Bottom) Significance of discovering CP violation. The red line is NOvA and T2K, the blue line is a $10 \mathrm{kton}$ LAr detector on-axis in the LBNE beam, and the green is the combination of those experiments. Solid black line is for CHIPS, from both a NuMI and LBNE run. Dotted lines show each experiment (or combination of experiments) without a CHIPS run. Solid lines show the effect of adding CHIPS to the results. 